\title{
Transient Tip-Sample Interactions in High-Speed AFM Imaging of 3D nano structures
}

\author{
Aliasghar Keyvani ${ }^{a}$, Hamed Sadeghian ${ }^{a}$, Hans Goosen, Fred van Keulen \\ Department of Precision and Microsystem Engineering, Delft University of Technology, Delft, \\ Netherlands; \\ ${ }^{a}$ Also at: Netherlands Organization for Applied Scientific research, TNO, Delft, Netherlands;
}

\begin{abstract}
The maximum amount of repulsive force applied to the surface plays a very important role in damage of tip or sample in Atomic Force Microscopy(AFM). So far, many investigations have focused on peak repulsive forces in tapping mode AFM in steady state conditions. However, it is known that AFM could be more damaging in transient conditions. In high-speed scanning, and in presence of 3D nano structures (such as FinFET), the changes in topography appear in time intervals shorter than the response time of the cantilever. In this case, the tip may crush into the sample by exerting much higher forces than for the same cantilever-sample distance in steady state situations. In this study the effects of steep upward steps in topography on the tip-sample interactions have been investigated, and it has been found that the order(s) of magnitude higher forces can be applied. The information on the worst case scenario obtained by this method can be used for selection of operation parameters and probe design to minimize damage in high-speed imaging. The numerically obtained results have been verified with the previous works in steady state regime. Based on this investigation the maximum safe scanning speed has been obtained for a case study.
\end{abstract}

Keywords: Tapping mode AFM, Tip-sample interactions, Peak repulsive Force, Hertz model, Contact stress

\section{INTRODUCTION}

Atomic Force Microscopy has already been suggested as one of the promising technologies for metrology and inspection of nanostructures for future semiconductor technologies. However, generally scanning probe technologies suffer from lack of speed and throughput for these applications. Thus fast and parallel operation is suggested to meet the requirements of industry. ${ }^{1,2}$ However, one of the important concerns in high-speed operation is that the probability of damaging the tip or the sample increases as the scanning speed increases.

The principle of atomic force microscopy is based on (exerting and) measuring interaction forces between sample surface and tip. Yet, depending on mechanical properties of the tip and the sample surface, excessive interaction forces can result in either tip wear, fracture or sample damage (hereafter shortly referred to as damage). Since the image quality is highly affected by the sharpness of the tip, the useful lifetime of the tip is limited by wear. Moreover, damage induces difficulties and unreliability in imaging soft samples. ${ }^{3,4}$

In general, damage at nanoscale can be attributed to different mechanisms such as fracture, plastic deformation or even chemical processes. ${ }^{5}$ Yet, all these processes can be considered primarily dependent on the stress in the contact region which is directly related to the applied force. ${ }^{5-8}$

The tip-sample forces in AFM mainly depend on the operation mode, e.g., while non-contact AFM works with weak attractive forces, contact mode AFM exerts strong repulsive and lateral forces.

Among different operation modes, the tapping mode is probably the most used one, due to its high-speed, stability and reduced damage. It has been devised to avoid constant contact and shear between tip and sample, and consequently, reduce the damage. However, even in tapping mode,there exist the risk of damage for imaging soft samples (e.g. biological samples) or in high-speed scanning. To control the damage, the probe, imaging parameters, and scanning speed should be selected according to sample properties. ${ }^{7,9}$ Moreover, in high-speed operation, abrupt changes in topography which occur within time intervals shorter than the response time of the cantilever increase the probability of damage. Especially in presence of steep steps (3D structures) in topography,

a.keyvanijanbahan@tudelft.nl

Metrology, Inspection, and Process Control for Microlithography XXIX, edited by Jason P. Cain,

Martha I. Sanchez, Proc. of SPIE Vol. 9424, 94242Q - () 2015 SPIE

CCC code: $0277-786 \mathrm{X} / 15 / \$ 18 \cdot$ doi: $10.1117 / 12.2185848$

Proc. of SPIE Vol. $942494242 Q-1$ 
of applied forces are much higher than what is foreseen for steady state condition.

One damage criterion in AFM is to compare the stresses in the contact area with the maximum allowable stress of the tip/sample material. ${ }^{4}$ Using the maximum repulsive force in each cycle and considering a contact mechanics model for the tip sample interaction, (e.g., Hertz, Tatara, Sneddon model or a Finite element model ${ }^{10-12}$ ) the maximum equivalent stress in the contact region can be estimated. This approach simplifies the nanoscale damage problem to calculation of the peak repulsive forces from the dynamics of the cantilever.

Unfortunately, the interaction forces in tapping mode are not directly accessible from measurements. ${ }^{13}$ For that reason, numerous analytic and numerical studies investigated the peak repulsive force in tapping mode AFM. For example, Guzman et al. ${ }^{14}$ numerically analyzed peak repulsive forces in an immersed AFM and studied differences between Tataras and Hertzian models for contact and also effects of material properties, e.g., viscoelasticity and fluid damping on peak repulsive force. As an example of analytical approaches, Wang ${ }^{15}$ used the Krylov-Bogolubov-Mitropolsky asymptotic method to solve for nonlinear dynamic motion of a cantilever under the influence of tip-sample interactions. Based on Wang's approach, Hu and Raman ${ }^{13}$ extracted an analytic solution for repulsive and attractive peak forces using periodic averaging method. Based on the analytic formulas presented by $\mathrm{Hu}$ and Raman, ${ }^{13}$ and considering the maximum stress criteria, Vahdat et al. ${ }^{7}$ introduced a method for selecting the imaging parameters.

The aforementioned studies provide comprehensive and practically applicable information on tip-sample interactions in steady state conditions. The steady-state assumption, which is a very fundamental assumption in previous studies, can introduce large errors when considering transient conditions. In literature, it is often assumed that the cantilever is vibrating with a certain amplitude while engaged to the surface. It is also assumed that only the first mode of the cantilever is excited and the other modes are completely neglected. ${ }^{11,13}$ For example, periodic averaging method consider linear vibration of the cantilever with a nonlinear deviation in the order of epsilon $O(\epsilon)$, that can change the amplitude and phase, while the motion remains harmonic. In this condition the amplitude is settled to the set point or the variations in amplitude and phase are so small and slow that their first derivative is assumed to be zero. ${ }^{13,16}$ However, it is well known that failure usually occurs while engaging the surface or in fast scanning conditions where the system is in a transient situation. This is specially true in situations in which the controller error is nonzero as well as the cantilever is not settled to a certain amplitude.

One example that causes the transient motion of the cantilever, happens when the probe suddenly encounters an upward change in topography (e.g., see Fig. 1). In that case not only the amplitude is not constant, but also the averaging methods are not applicable anymore. Moreover, it will be experimentally and theoretically shown in this paper that the one degree of freedom (1-DOF) oscillator model for the cantilever (which is the other limiting assumption in most literature ${ }^{17}$ ) fails to represent the transient behavior of the cantilever.

Based on the requirements of semiconductor industry and demand for high-speed AFM a thorough theory is needed to investigate and control the damage, considering the transient conditions. In order to address transient behavior of cantilever, this paper investigates the tip-sample interactions when a sudden change in topography appears. This study can cover the effects of very high-speed scanning to estimate the scanning speed limit from damaging point of view.

The present paper is organized as following: the next section presents a dynamic model of the cantilever using Derjaguin-Muller-Toporov(DMT) tip-sample interaction model ${ }^{11}$ and compares the peak repulsive force in the steady state regime with the analytic formula as presented by Hu and Raman. ${ }^{13}$ Section III presents a numerical investigation of steep upward changes in topography. Section IV contains a case study where the maximum scanning speed has been found for tapping mode imaging of a FinFET structure. The conclusions are presented in the last section.

\section{STEADY STATE ANALYSIS}

In Tapping mode AFM, the cantilever is excited with a frequency $(\omega)$ closed to its fundamental resonance frequency $\left(\omega_{n}\right)$ using a dithering piezo actuator while it is in the air and is brought close to the sample surface using a $\mathrm{z}$ positioning actuator. Due to the Tip-sample interactions the vibration amplitude of the cantilever reduces from the free air amplitude $\left(A_{i}\right)$ by a factor $A_{r}$ (so-called amplitude ratio) to an engaged amplitude 


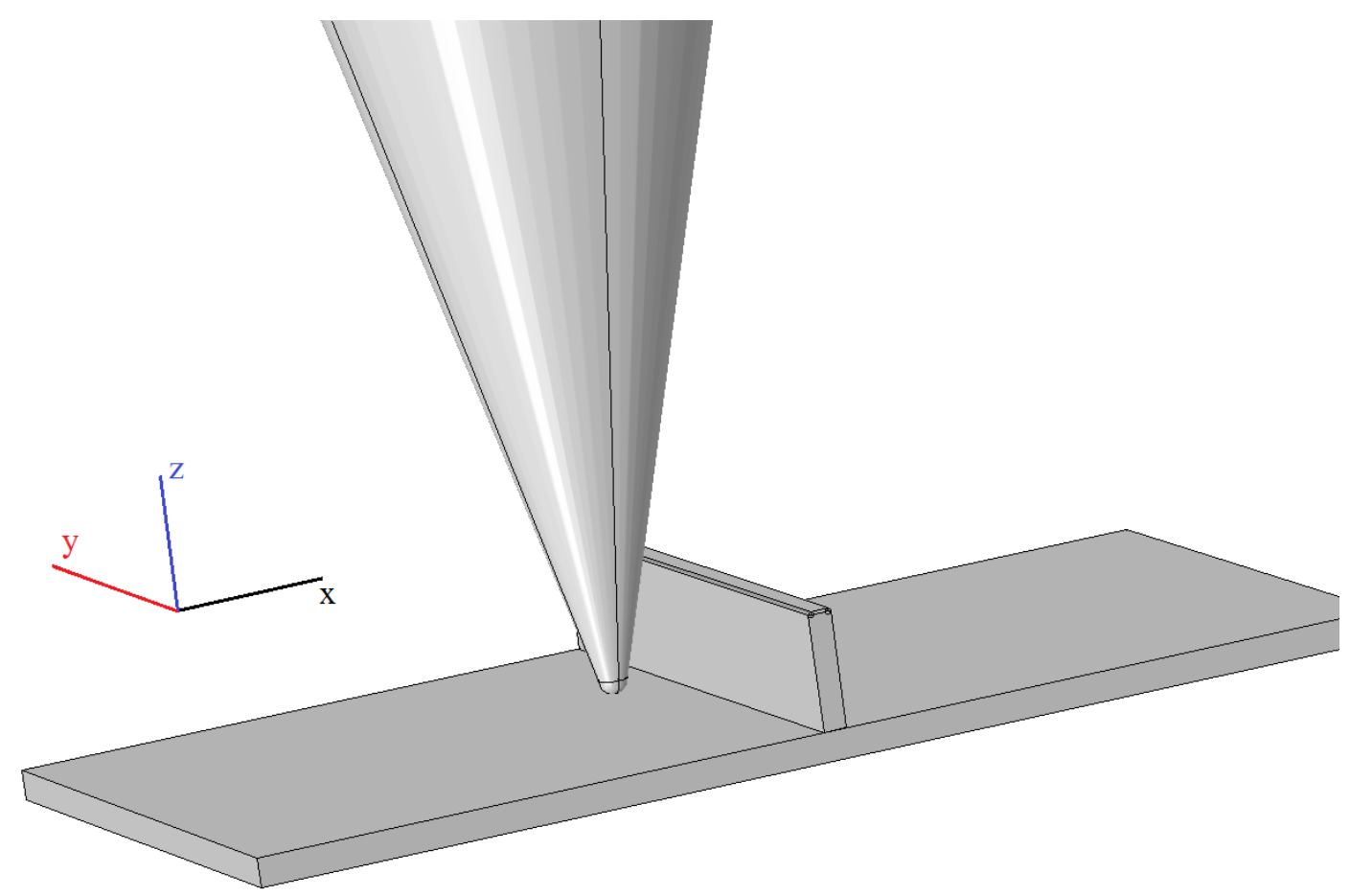

Figure 1. Schematic view of tip and surface with a 3D nano structure

( $\left.A=A_{i} A_{r}\right)$. The role of the controller is to keep the amplitude ratio constant by adjusting the position of cantilever or the surface with respect to each other while scanning in $\mathrm{x}-\mathrm{y}$ direction. For steady state conditions of a rectangular cantilever in air(or vacuum), the motion of a cantilever can be approximated using a lumped one DOF mass spring model which is excited around its fundamental resonance frequency. Considering Hertz's model for contact area and attractive van der Waals (vdW) forces (that resemble the DMT model ${ }^{18}$ ) and an equivalent dither piezo excitation force $\left(F_{0}\right)$, the governing equation for dynamic motion of the tip can be expressed as:

$$
m \ddot{x}+c \dot{x}+k x=\frac{\eta_{a} R}{6\left(g_{0}-x\right)^{2}}-\frac{4}{3} E_{e f f} \sqrt{R} \delta^{3 / 2}+F_{0} \sin (\omega t)
$$

where $x$ represents the displacement of the tip, $k, c, m, R$ and $\eta_{a}$ are spring constant, damping ratio, effective mass of cantilever, tip radius and Hamaker constant, respectively. The distance between un-deflected position of tip and sample surface is defined as $g_{0}$. The indentation between sample and the tip is defined as $\delta=$ $\left(a_{0}+x-g_{0}\right) \mathrm{H}\left(a_{0}+x-g_{0}\right)$ in which $a_{0}$ is the atomic separation distance where the Heaviside function $\left(\mathrm{H}\left(a_{0}+x-g_{0}\right)\right)$ is used to indicate that indentation is zero when the tip is not in contact with the sample. The effective stiffness of the surface, $E_{e f f}$ is given as $\frac{1}{E_{e f f}}=\frac{1-\nu_{t}^{2}}{E_{t}}+\frac{1-\nu_{s}^{2}}{E_{s}}$, where $E$ and $\nu$ are young's modulus and poisson's ratio and subscript $t$ and $s$ refer to tip and sample respectively. ${ }^{11}$

$\mathrm{Eq}(1)$ was solved using a $4^{\text {th }}$ order Rung-Kutta integration scheme. Fig. 2 shows the tip position in time domain and accompanying tip-sample force for a cantilever which properties are demonstrated in the caption.

To verify the numerical method, we compare the results with the analytic solution of Hu and Raman. ${ }^{13}$ They presented an equation showing the peak repulsive force for different amplitude ratios. Although the amplitude and free air amplitude do not explicitly appear in $\mathrm{Eq}(1)$, we can obtain the forces in terms of amplitude ratio by running the simulation for different amplitudes and amplitude ratios. Fig. 3 shows peak repulsive force in contact considering different tip radii.

As can be seen from Fig. 3, the numerical results show a similar trend as the analytic results. The biggest discrepancy occurs when the tip radius is high and the amplitude is low. The reason for the discrepancies is that 

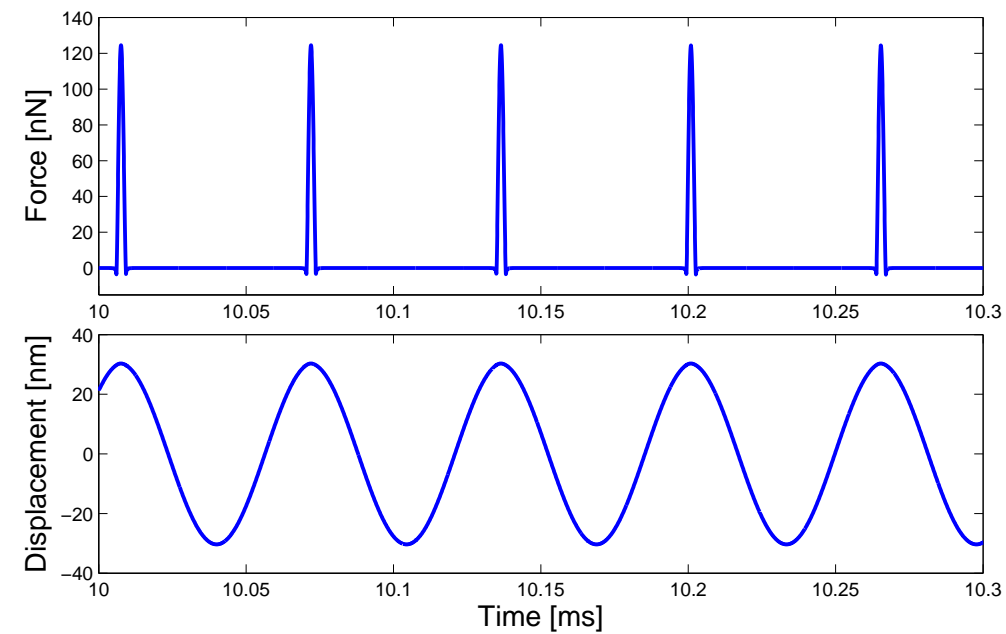

Figure 2. Tip-sample interaction and tip displacement for a cantilever with a spring constant of $k=6 \mathrm{~N} / \mathrm{m}$ Q factor 50 , tip radius of $R=10 \mathrm{~nm}$, free air amplitude of $A_{i}=50 \mathrm{~nm}$, resonance and working frequency of $\omega_{n}=100 \mathrm{kHz}$, and amplitude ratio of $A_{r}=90 \%$

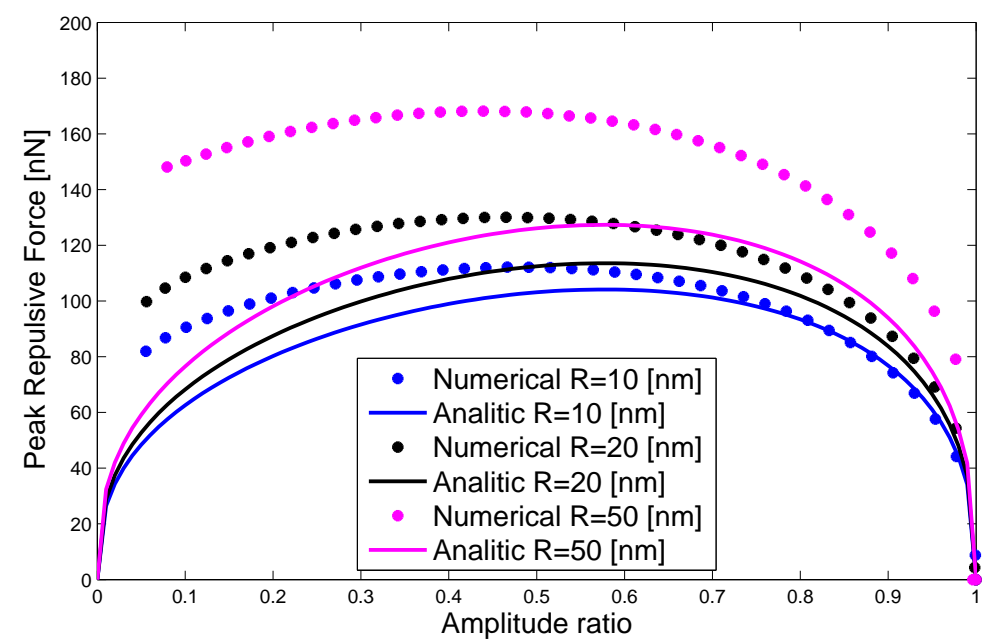

Figure 3. Peak repulsive force for a cantilever with spring constant of $6 \mathrm{~N} / \mathrm{m}$, Q factor 50 , free air amplitude of $50 \mathrm{~nm}$, and resonance frequency of $100 \mathrm{kHz}$, with different tip-Radii and obtained from solution of Eq(1) and analytic method. ${ }^{13}$ 


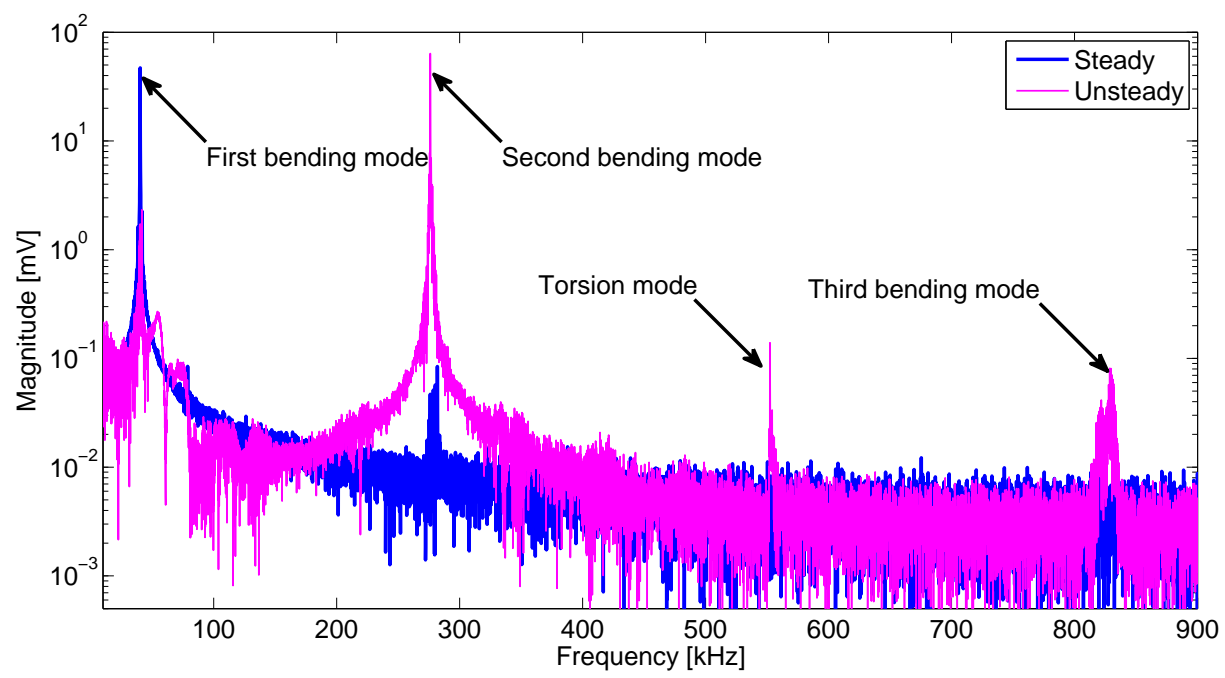

Figure 4. Motion spectra of the cantilever. free air amplitude is $1 \mathrm{~V}$ and the cantilever is only excited in its first resonance frequency $(\approx 41 \mathrm{kHz})$

the analytic expression for peak repulsive forces as presented by $\mathrm{Hu}$ and Raman ${ }^{13}$ is obtained considering only repulsive forces (i.e. ignoring the attractive $\mathrm{vdW}$ forces). Ignoring the $\mathrm{vdW}$ forces leads the overall forces to be underestimated and snap-in phenomenon to be ignored. The snap-in phenomenon happens while the amplitude is excessively low and the radius of the tip is notably large, causing the vdW forces to become more dominant. At a certain amplitude the restoring elastic force of the cantilever can not overcome the vdW forces. This causes the tip to snap to the surface and stick there.

\section{TRANSIENT SITUATIONS}

There can be different sources that can cause transient situation in AFM. However, it is not practically possible to investigate all their effects on the applied force. In this section a steep upward step in topography is used to show the effect. Experimental results in this section suggest that, the one DOF model for the cantilever is not sufficient for modeling the cantilever in transient conditions and numerical results show that, transient conditions can be an order of magnitude more damaging.

\subsection{One DOF Assumption}

As it is mentioned above, the one DOF assumption only holds for single frequency Amplitude Modulation (Am) mode imaging with normal probes in air, for example it does not hold for special harmonic probes, ${ }^{19}$ or for multi-modal AFM ${ }^{20}$ neither for the transient condition. In order to experimentally demonstrate this fact, a commercially available rectangular cantilever (MP-P22120 Bruker) is utilized to image a flat silicon surface while the controller gains are deliberately chosen to be unstable so that the amplitude is not settled.

Fig. 4 shows the frequency spectrum of motion of the cantilever. it demonstrates that higher modes of cantilever are excited considerably due to fluctuations of amplitude. It can be concluded that one DOF oscillator assumption for the cantilever results in a large error.

\subsection{Sudden Changes in Topography}

During the coarse approach of probe towards sample or while scanning fast, the tip can hit the surface much faster than in steady state conditions. Thus, it applies a much higher repulsive force on the surface. The applied force in transient situation is very challenging to measure, because of two reasons. First, the forces in tapping mode are not directly accessible via experiments and second, it happens in a very short interval of time. On the other hand, it can not be calculated with present analytic techniques, because it is not steady. 


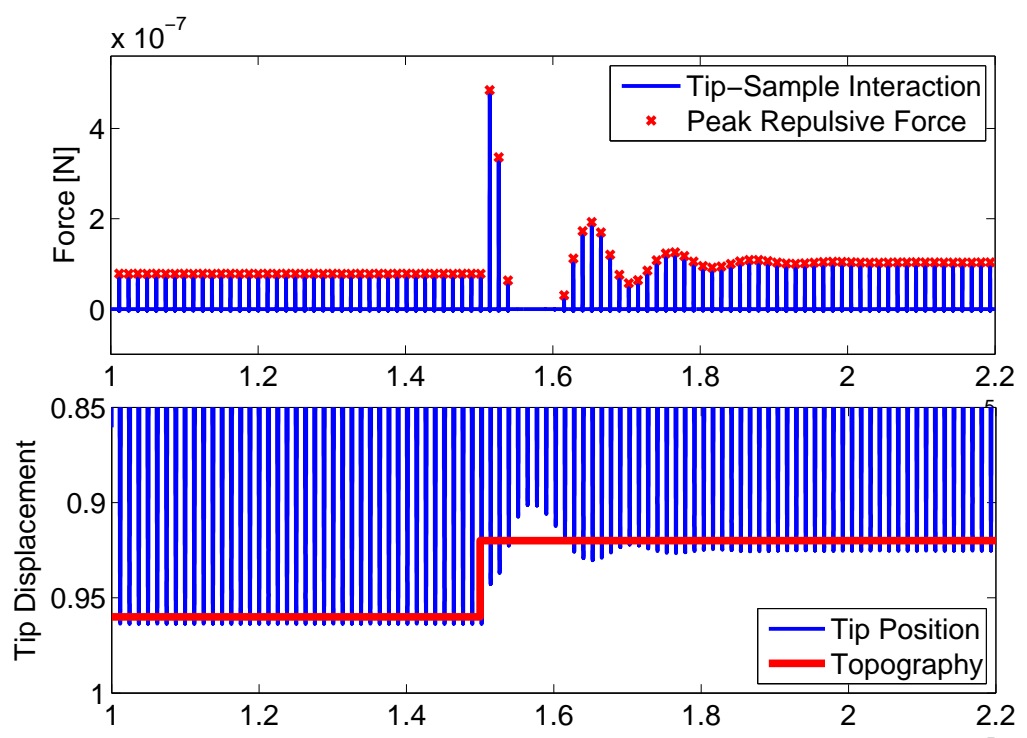

Figure 5. Demonstration of sudden change in topography for a cantilever with spring constant of $5 \mathrm{~N} \mathrm{~m}^{-1} \mathrm{Q}$ factor 50 tip radius of $10 \mathrm{~nm}$ free Air amplitude of $50 \mathrm{~nm}$ resonance and working frequency of $100 \mathrm{kHz}$. it has been assumed that suddenly a $2 \mathrm{~nm}$ ( $4 \%$ of initial amplitude) step appears in front of tip.

It thus violates the periodicity and one DOF oscillator assumption. However, the numerical solution of the full nonlinear partial differential equation governing the cantilever motion can demonstrate the phenomenon. Fig. 5 shows the interaction forces and non-dimensional tip position with respect to time that is found by numerically solving the partial differential equation of the cantilever considering DMT model. Note that for the sake of clear demonstration, ideally high amount of damping $(\mathrm{Q}=50)$ is considered to eliminate the ambiguous fluctuations. As can be seen from Fig. 5, order(s) of magnitude higher forces can be applied in unsteady situations, making it very important to investigate the phenomenon. However, it is not always easy to solve the full nonlinear PDE numerically and for every different situations. Here we present a closed form solution based on energy conservation law at the goal of finding the amount of maximum force in terms of size of the steep upward step, amplitude, frequency, spring constant, etc.

\section{CASE STUDY}

One situation that a sudden upward step can appear in front of tip, is in high-speed inspection or imaging of Fin Field Effective Transistors (FinFET) in semiconductor industries. They consist of a thin silicon fin (as source, conducting channel and drain), wrapped with the gate electrode that show better performance as transistors. Depending on the scanning speed this structures can cause a sudden step effect for the tip. Here we consider the effect of scanning velocity in worst case scenario from damaging point of view. Consider that the tip is approaching the nano structure with a lateral velocity of $v$ while its is vibrating in tapping mode with an amplitude of $A$, frequency $\omega$ and free air amplitude $A_{i}$, then the conical part of the tip will engage the corner of the nano-structure. Then the effective size of sudden step in topography in worst case scenario can be calculated as $h=v \frac{2 \pi}{\omega} \cot (\alpha)$, where $\alpha$ is the angle of the tip. As a case study we consider the operation parameters and probe characteristics as indicated in Table 1. and we seek for the scanning velocity limit using presented method.

Considering different step heights for the sudden change effects Fig. 6 shows the forces in time domain simulated for the parameters mentioned in Table1. For this specific case study, fitting the maximum amount of Peak repulsive forces with respect to step height, the maximum safe scanning velocity can be found as a function of allowable force as:

$$
v_{\max }=\frac{\omega}{2 \pi} \tan (\alpha)\left(-0.474+1.46 \times 10^{-2} F_{M}+1.9 \times 10^{-3} F_{M}^{2}\right)
$$

where $F_{M}$ is the maximum allowable force in nano Newton, $\frac{\omega}{2 \pi}$ is the working frequency in $\mathrm{Hz}$ and the resultant velocity would be in $\mathrm{nm} \mathrm{s}^{-1}$. 
Table 1. Dynamic properties of the probe and AFM operation parameters

\begin{tabular}{ll|ll}
\hline \hline \multicolumn{1}{c}{ Spring constant } & $0.2 \mathrm{~N} / \mathrm{m}$ & Q factor & 100 \\
\hline Resonance frequency & $100 \mathrm{kHz}$ & Tip-radius & $10 \mathrm{~nm}$ \\
\hline Young Modulus (for both) & $130 \mathrm{GPa}$ & Poisson ratio & 0.3 \\
\hline Free air amplitude & $30 \mathrm{~nm}$ & Set point amplitude & $20 \mathrm{~nm}$ \\
\hline Tip cone angle & $26^{\circ}$ & Excitation frequency & $99.5 \mathrm{kHz}$ \\
\hline
\end{tabular}

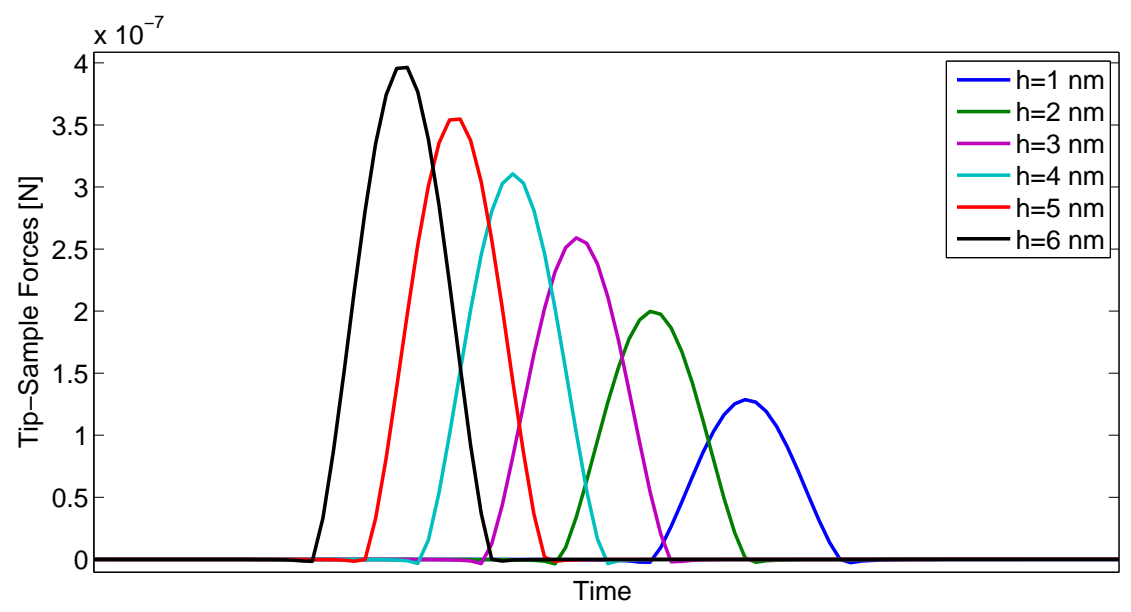

Figure 6. Tip-sample interactions in time domain considering different step height in sudden contact

In order to calculate the maximum allowable force the present case study, a finite element model (Comsol 4.4) was used. Fig. 7 shows the von Mises stress in contact area for this case study. Here the conical part of the tip is in contact with the corner of the FinFet structure. Using a parametric sweep in the finite element model presented in Fig. 7 the amount of maximum von Mises stress can be found in terms of applied repulsive force(Fig. 8).

As a damage criterion it would be relevant to compare the maximum stress in contact area with maximum allowable stress for the material. With this assumption the maximum permissible stress for the material is related to the hardness of the material. For example the maximum permissible contact stress for silicon and silicon-nitride are about $6 \mathrm{GPa}$ and $12 \mathrm{GPa}$ respectively. ${ }^{4}$ In this case study we assumed that both the tip and sample are made from silicon. Thus, considering Fig. 8 it can be concluded that the maximum permissible peak repulsive force for this geometries is about $40 \mathrm{nN}$.

So according to the finite element model for this specific case study the forces bigger than $40 \mathrm{nN}$ would cause damaging stresses in contact area. On the other hand according to $\mathrm{Eq}(2)$ that is a fitting result of dynamics of beam, the maximum safe scanning velocity can be calculated as $160 \mu \mathrm{m} \mathrm{s}^{-1}$ Note that the $\mathrm{Eq}(2)$ is valid only for specified parameters in Table1. and generalizing the results is a remaining challenge.

\section{CONCLUSION}

In this paper the tip-sample forces were calculated with and without considering the sudden changes in topography. It was shown that presence of steep upward steps in topography can significantly increase the applied forces. The numerical results were compared with analytic methods from literature and their validity was verified. Then contact forces and stresses calculated for sudden changes in topography, used together with a finite element model for finding the scanning speed limit from damaging point of view. 


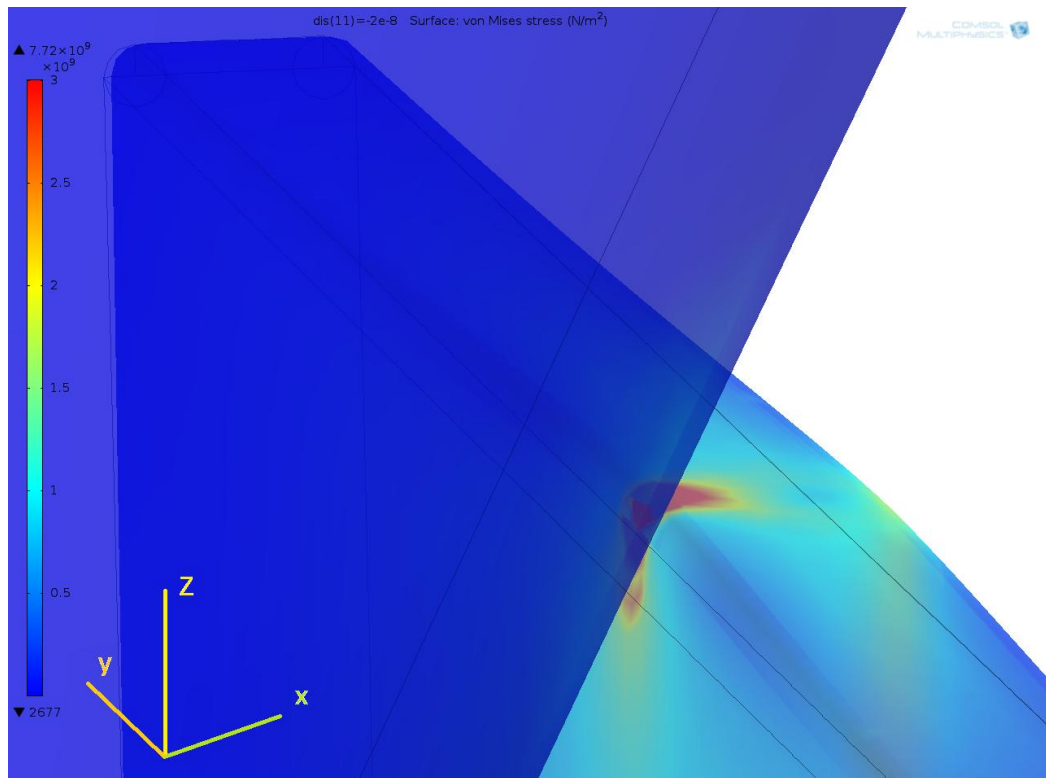

Figure 7. von Mises stress distribution on FinFet structure of $100 \mathrm{~nm}$ height, $14 \mathrm{~nm}$ and edge fillet radius of $1 \mathrm{~nm}$ in contact with AFM tip with an apex angel of $1 \mathrm{~nm}$.

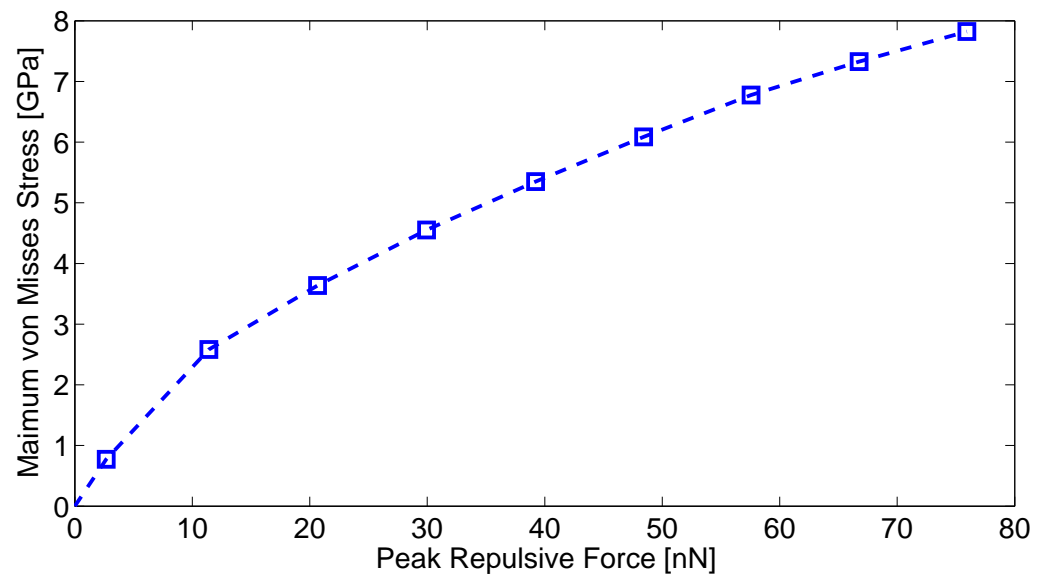

Figure 8. Maximum von Mises stress vs. applied repulsive force for the present case study 


\section{acknowledgment}

This works was supported by Netherlands Organization for Applied scientific Research, TNO, Early Research Program 3D Nanomanufacturing Instruments.

\section{REFERENCES}

[1] H. Sadeghian, T. C. van den Dool, W. E. Crowcombe, R. W. Herfst, J. Winters, G. F. I. J. Kramer, and N. B. Koster. Parallel, miniaturized scanning probe microscope for defect inspection and review. 9050:90501B, 2014.

[2] H Sadeghian, N B Koster, and T C van den Dool. Introduction of a high throughput SPM for defect inspection and process control. Proc. SPIE 8681, Metrology, Inspection, and Process Control for Microlithography XXVII, 8681(Figure 1):868127-8, 2013.

[3] Carlo a Amadei, Tzu Chieh Tang, Matteo Chiesa, and Sergio Santos. The aging of a surface and the evolution of conservative and dissipative nanoscale interactions. The Journal of chemical physics, 139(8):084708, August 2013.

[4] M.L. Bloo, H. Haitjema, and W.O. Pril. Deformation and wear of pyramidal, silicon-nitride AFM tips scanning micrometre-size features in contact mode. Measurement, 25(3):203-211, April 1999.

[5] Tevis D B Jacobs and Robert W Carpick. Nanoscale wear as a stress-assisted chemical reaction. Nature nanotechnology, 8(2):108-12, February 2013.

[6] Koo-Hyun Chung, Yong-Ha Lee, and Dae-Eun Kim. Characteristics of fracture during the approach process and wear mechanism of a silicon AFM tip. Ultramicroscopy, 102(2):161-71, January 2005.

[7] Vahid Vahdat, Robert W Carpick, Mechanical Engineering, Applied Mechanics, and United States. Practical Method to Limit Tip À Sample Contact Stress and Prevent Wear in. (11):9836-9850, 2013.

[8] San Diego and La Jolla. Nano-wear of the diamond AFM probing tip under scratching of silicon, studied by AFM. 2:345-354, 1996.

[9] Justin Legleiter. The effect of drive frequency and set point amplitude on tapping forces in atomic force microscopy: simulation and experiment. Nanotechnology, 20(24):245703, June 2009.

[10] Hamid Ladjal, Jean Luc Hanus, Anand Pillarisetti, Carol Keefer, Antoine Ferreira, and Jaydev P. Desai. Atomic force microscopy-based single-cell indentation: Experimentation and finite element simulation. 2009 IEEE/RSJ International Conference on Intelligent Robots and Systems, IROS 2009, pages 1326-1332, 2009.

[11] Horacio V Guzman, Alma P Perrino, and Ricardo Garcia. Peak forces in high-resolution imaging of soft matter in liquid. ACS nano, 7(4):3198-204, April 2013.

[12] S. a. Chizhik, Z. Huang, V. V. Gorbunov, N. K. Myshkin, and V. V. Tsukruk. Micromechanical properties of elastic polymeric materials as probed by scanning force microscopy. American Chemical Society, Polymer Preprints, Division of Polymer Chemistry, 39(15):1146-1147, 1998.

[13] Shuiqing Hu and Arvind Raman. Analytical formulas and scaling laws for peak interaction forces in dynamic atomic force microscopy. Applied Physics Letters, 91(12):123106, 2007.

[14] Horacio V Guzman and Ricardo Garcia. Peak forces and lateral resolution in amplitude modulation force microscopy in liquid. Beilstein journal of nanotechnology, 4:852-9, January 2013.

[15] Lugen Wang. Analytical descriptions of the tapping-mode atomic force microscopy response. Applied Physics Letters, 73(25):3781, 1998.

[16] S.H. Strogatz. Nonlinear Dynamics and Chaos: With Applications to Physics, Biology, Chemistry, and Engineering. Advanced book program. Westview Press, 1994.

[17] Robert Stark, Georg Schitter, Martin Stark, Reinhard Guckenberger, and Andreas Stemmer. State-space model of freely vibrating and surface-coupled cantilever dynamics in atomic force microscopy. Physical Review B, 69(8):085412, February 2004.

[18] B.V Derjaguin, V.M Muller, and Yu.P Toporov. Effect of contact deformations on the adhesion of particles. Journal of Colloid and Interface Science, 53(2):314-326, November 1975. 
[19] Ozgur Sahin, Sergei Magonov, Chanmin Su, Calvin F Quate, and Olav Solgaard. An atomic force microscope tip designed to measure time-varying nanomechanical forces. Nature nanotechnology, 2(8):507-14, August 2007.

[20] Ricardo Garcia and Elena T Herruzo. The emergence of multifrequency force microscopy. Nature nanotechnology, 7(4):217-26, April 2012. 